\title{
Performance of OFDM in High-Mobility Environments
}

\author{
Robert Thrasher and Greg Pottie
}

\begin{abstract}
This paper focuses on communications within a high-mobility context with OFDM as the modulation of choice. We first show that the Air-to-Ground (AtG) channel is the most difficult to adapt using standard channel equalization methods. We then derive a new channel model (AtGMM) for the AtG case and use it as the basis for analysis and for simulating the effects within the AtG environment. Next, we derive the optimal channelestimation-based equalizer for the AtG channel; showing performance equivalent to a comparable time-invariant channel. We then analyze the performance of conventional OFDM-based systems using the AtGMM channel model and provide guidelines to maximizing the throughput within differing mobility contexts.
\end{abstract}

Keywords: O FDM, fast fading, equalization.

\section{INTRODUCTION}

Orthogonal frequency division multiplexing (OFDM) [1] has received considerable attention over the years owing to the relative simplicity of its adaptation to dispersive channels. Indeed, the ability of OFDM to, in effect, "transform" a dispersive wireless channel to a Gaussian one makes it an attractive choice when it is combined with other modulation strategies. Some notable marriages are multi-carrier code-division-multiple-access 2 (MC-CDMA) and multiple input/multiple output OFDM [3] (MIMO-OFDM). In the case of MC-CDMA, OFDM enables one to harness diversity [4 in combating frequency-selective fading when symbols are spread across widely separated sub-carriers. MIMO, in its simplest form, requires a flat channel response [5] which can be easily provided by selecting sufficiently narrow OFDM sub-channels.

\subsection{Internet-in-the-Sky}

It is to this end that OFDM is being employed in a research project at UCLA. Our vision is to create an "Internetin-the-sky" to support communication needs in remote areas where wired network access is not available. Fig. 1 illustrates the "hierarchical" network envisioned. The hierarchy imposed here is such that nodes with access to greater information bandwidth can serve as gateways for communication to nodes within other clusters that might be accessible only at high cost or not at all. With regard to a wireless "Internet-in-the-sky" concept, this implies that the higher altitude nodes should be the more capable ones. Because OFDM is one of the central components of this effort, special emphasis has been focused on understanding the impairments and the corresponding mitigating strategies relevant to OFDM.

Section 1.2 offers a brief review of OFDM. Recognizing that the Air-to-Ground (AtG) channel presents both high doppler and time dispersion (a challenging equalization combination), in section 2 we derive a new channel model to describe the AtG channel replacing the traditional time-invariant tapped delay line channel model. Using this new dynamical model of the AtG channel, section 3 shows that channel estimation-based equalization in the time-domain provides more responsive tracking of channel dynamics and eliminates intercarrier interference (ICI). Since section's 3 equalization is complex compared to conventional OFDM, we consider the performance of conventional OFDM using the AtG channel model derived in section 2 to derive, in section 4, the SINR under this new channel model. We compare the theoretical performance with simulation and also present the simulated performance of the optimal equalization obtained in section 3 . Finally, in section 5$]$ we show that the throughput of OFDM (under a constant BER constraint) under dynamic channel conditions is limited by cyclic prefix overhead for low RMS frequency offsets whereas, at high RMS frequency offsets it is limited by the ICI.

The authors are with the Electrical Engineering Department, University of California, Los Angeles, USA. Contact Info: Email: BobThrasher@cox.net, pottie@icsl.ucla.edu 


\subsection{OFDM Overview}

Consider an OFDM system having $\mathrm{N}$ subcarriers and a cyclic prefix length of L. Each OFDM symbol, $i$, is formed as the inverse discrete Fourier transform (IDFT) of a block of $\mathrm{N}$ data symbols $S_{i}$ concatenated to a block of $\mathrm{L}$ samples taken from the tail of the prior IDFT output sequence. The result is passed through a pulse shaping filter, $p_{n}$, to yield the following transmitted sequence:

$$
x_{n}=\sum_{i=-\infty}^{\infty} s_{i, n-i} p_{n-i}
$$

where $s_{i}$ is the IDFT of the $i^{\text {th }}$ block of data symbols, $S_{i}$, extended by a cyclic prefix:

$$
s_{i, k}= \begin{cases}\sqrt{\frac{1}{N}} \sum_{l=0}^{N-1} S_{i, l} e^{j 2 \pi l k / N}, & \text { for } 0 \leq k<N+L \\ 0, & \text { otherwise }\end{cases}
$$

When the sequence $x_{n}$ is passed through a linear time-invariant (LTI) channel $h_{n}$ of length $g$, such as is depicted in Fig. 2, inter-symbol interference (ISI) will occur between adjacent OFDM symbols. This paper assumes that $g<L$ by design. The received signal is:

$$
y_{n}=\sum_{j=-\infty}^{n} x_{j} h_{n-j}+\nu_{n}
$$

where $\nu_{n}$ is assumed to be white Gaussian noise (WGN) ( $\nu_{n}$ will be used loosely in the following to represent noise). If we assume perfect timing synchronism, then at the receiver a set of $\mathrm{N}+\mathrm{L}$ signal samples is taken corresponding to the $i^{t h}$ OFDM symbol. By discarding the first $L$ samples the cyclic prefix is removed. Using array notation, we can describe this remaining block of $N$ samples as:

$$
Y_{i}=C_{n} H C_{p} W^{H} S_{i}+\nu_{i}
$$

where $W$ is the N-by-N DFT matrix, $C_{p}$ is the $\mathrm{N}+\mathrm{L}-$ by- $\mathrm{N}$ cyclic prefix matrix, $C_{n}$ is the N-by-N+L cyclic removal matrix, and $\mathrm{H}$ is the $\mathrm{N}+\mathrm{L}-$ by- $\mathrm{N}+\mathrm{L}$ Toeplitz channel matrix (Toeplitz for time-invariant channels). The resulting block, $Y_{i}$, is processed by performing the DFT:

$$
R_{i}=W C_{n} H C_{p} W^{H} S_{i}+\nu_{i}=W C W^{H} S_{i}+\nu_{i}
$$

where the N-by-N matrix, $C=C_{n} H C_{p}$, has the circulant property which implies that $W C W^{H}$ is diagonal.

Since $g<L$, the ISI from the $i-1^{\text {th }}$ symbol does not extend further than the length of the (discarded) L-sample guard interval of the $i^{t h}$ symbol. This leads to OFDM's desirable single-tap equalization characteristic. And further, the circulant property of $C$ effectively leads to a decoupling of the equations in (3) so that the equalization coefficients may be computed with complexity $O(N)$.

\subsection{OFDM and Fast Fading}

In the case of mobile systems, the issue of primary concern is fading-rate. There are a number of trends that have forced communications designers to accommodate ever-higher fading rates. First, the scarcity of radio spectrum at the low end is forcing consideration of higher frequency bands. In turn fading rates are higher - proportional to the increased carrier frequency. Second, since the present research seeks a solution that is applicable across a wide range of vehicle mobilities (from stationary to high dynamic aircraft), provision must be made for the most extreme fading case. Third, for block oriented modulations such as OFDM, the fading rate is proportional to the block length.

In fast fading environments, the least-mean-squares (LMS) algorithm may not be able to adapt to the rapid channel variations. Recursive least-squares (RLS) adaptation may be required to remedy this at the expense of an order of magnitude complexity increase. Additionally, the choice of adaptation domain may have an effect on the tracking speed of a particular adaptation algorithm. This is where channel estimation based adaptation may help since this domain is often described by fewer parameters than in the equalizer-tap domain. 


\section{THE AIR-TO-GROUND MULTIPATH MODEL (ATGMM)}

The one factor that virtually all of the adaptation methods up to the present time have in common is that they treat the channel or equalization coefficients as either random or unknown constants. This situation derives from using a channel model such as is depicted in Fig. 2. More specifically, it is assumed that the time evolution of the process cannot be described by some time-propagation model and therefore is treated as random. For many environments, the assumption of an unpredictable evolution of parameters is reasonable. Such fading environments are characterized by their doppler power spectrum. But, what if the parameter evolution could be accurately described using some dynamical model? In principle, tracking such a parameter set should be less demanding than the traditional non-dynamical set (in the presence of dynamics). This principle is well established in controls where higher-order tracking algorithms exhibit less tracking lag if the underlying process is of a compatible order. In this section we take a detailed look at the air-to-ground (AtG) channel and propose a new channel model to describe it.

We begin by first reproducing a continuous-time model of the multipath environment 6 .

$$
c(\tau ; t)=\sum_{m} \alpha_{m}(t) e^{j 2 \pi f_{c} \tau_{m}(t)} \delta\left(\tau-\tau_{m}(t)\right)
$$

This relation expresses the time-variant impulse response of a continuous-time channel as it evolves in time $t$ with a number of arrivals at delays $\tau_{m}(t)$. We note that the model is expressed in terms of a superposition of discrete arrivals with each arrival $m$ having a particular amplitude $\alpha_{m}(t)$ and phase $e^{j 2 \pi f_{c} \tau_{m}(t)}$. Since the delay $\tau_{m}$ is the ratio of range $R_{m}$ to the speed of light $c$, we have the following alternate expression:

$$
c(\tau ; t)=\sum_{m \in M(t)} \alpha_{m}(t) e^{j 2 \pi R_{m}(t) / \lambda_{c}} \delta\left(\tau-R_{m}(t) / c\right)
$$

where $\lambda_{c}$ is the carrier wavelength and the set of arrivals at time $t$ and any delay $\tau$ we denote as $M(t)$. Now that we have emphasized in (4) the fact that multipath is range-dependent, we can proceed to examine the effect of mobility within various environments. The environments we have identified for this research are the following: 1) Air-to-Air (AtA), 2) Ground-to-Ground (GtG), 3) Air-to-Ground (AtG).

The GtG environment is characterized by doppler consistent with ground vehicle speeds and significant timedispersion caused by nearby scatterers. The AtA environment (i.e. antennae not near the ground) is characterized by high doppler and a singular line-of-sight (LOS) ray with negligible received reflected rays. The AtG case is characterized by high doppler and substantial dispersion caused by scatterers nearby the ground antenna. We also note that there may be several of these dispersion "clusters" of received energy due to the presence of large reflectors that are distant with respect to the receiver and transmitter. The three scenarios are depicted in Fig. 3 .

\subsection{Air-to-Air (AtA)}

The AtA case is clearly the simplest of the three and can be considered a non-fading flat channel. For this to be reasonable the ground reflection must be insignificant and the transceiver implementation must employ carrier synchronization. In this case, (4) collapses to the following:

$$
c(\tau ; t)=\alpha(t) e^{j 2 \pi R(t) / \lambda_{c}} \delta(\tau-R(t) / c)
$$

Focusing on the only impulse arrival at delay $\tau=R(t) / c$ and expressing the range to the first order as $R(t)=$ $R_{0}+\dot{R} t$, we have:

$$
c(t)=\dot{\alpha}(t) e^{j 2 \pi \dot{R} t / \lambda_{c}}
$$

where we have absorbed the phase constant $e^{-j 2 \pi R_{0} / \lambda_{c}}$ into $\alpha(t)$. Finally, to further simplify the AtA model, we can treat the complex gain $\alpha(t)$ as constant as compared to the time-variant phase rotation $e^{j 2 \pi \dot{R}(t) / \lambda_{c}}$ to yield:

$$
c(t)=\alpha e^{-j 2 \pi \dot{R} t / \lambda_{c}}
$$




\subsection{Ground-to-Ground (GtG)}

To proceed for the GtG case, we return to (4) and focus on the $m^{t h}$ multipath ray. Next make the assumption that all signal reflectors are physically stationary with respect to the antennas. We can then conclude that the range rate of a given multipath ray is the following linear combination of the velocities of the transmitter and receiver antennas:

$$
\dot{R}_{m}=\dot{R}_{m, r}+\dot{R}_{m, t}=V_{r} \cdot L_{m, r}+V_{t} \cdot L_{m, t}
$$

which says that the total range rate is the sum of the receiver velocity projected onto the LOS of the multipath ray arriving at the receiver plus the same corresponding to the ray departing the transmitter. Since we are focusing on the $m^{\text {th }}$ multipath ray, our GtG model, adapted from (5), is now:

$$
c\left(\tau_{m}, t\right)=\alpha_{m}^{\prime} e^{j 2 \pi\left(V_{r} \cdot L_{r, m}+V_{t} \cdot L_{t, m}\right) t / \lambda_{c}}
$$

From (7) we can arrive at the discrete-time channel impulse response:

$$
h_{k, n}=\sum_{m \in \dot{M}(k, n)} c\left(\tau_{m}, n T_{s}\right) e^{-j 2 \pi \dot{R}_{m}\left(\tau_{m}\left(n T_{s}\right)-k T_{s}\right) / \lambda_{c}}
$$

where we have "clustered" the arrivals corresponding to the $k_{t h}$ delay accordingly:

$$
\dot{M}(k, n) \equiv\left\{m: k T_{s} \leq \tau_{m}\left(n T_{s}\right)<(k+1) T_{s}\right\}
$$

If there are a large number of scatterers nearby either antenna, then it is clear that the distribution of $\left|h_{k, n}\right|$ will be Rayleigh [7.

\subsection{Air-to-Ground (AtG)}

The relation expressed in (8) can now be used to aid our understanding of the AtG case. Since the complex exponential changes slowly with time it will not contribute to fading-rate and so we may absorb it into $\alpha_{m}$ :

$$
h_{k, n}=\sum_{m \in M(k, n)} \alpha_{m}^{\prime} e^{j 2 \pi n T_{s}\left(V_{r} \cdot L_{r, m}+V_{t} \cdot L_{t, m}\right) / \lambda_{c}}
$$

To continue, we require some assumptions. First, in the AtG case, we can safely assume that the signal emanating from the airborne vehicle does not encounter any local scattering as in the case of the ground vehicle. Second, there may be one or more large scatterers, such as mountains, that are assumed distant with respect to the airborne vehicle. Finally, we shall assume that the signals reflected from our large scatterers are clustered together in delay and that these clusters are not overlapping in time at the receiver. In other words, the range of delays due to all multipaths received from one "mountain" will not overlap the group received from a second "mountain". Of course, it is possible that these groups of multipath arrivals may overlap in delay, but generally this will not be the case. On account of these assumptions we can further specialize our channel model.

In order to visualize this situation refer to Fig. 3. The assumptions previously mention provide the following. The first two assumptions taken together provide that the departing multipath rays from the airborne antenna which are reflected by one of the distant scatterers will be nearly parallel. So we may assume that the angles between these rays and the aircraft's velocity vector are nearly equal. The last assumption provides that for a given delay index $k$, all of the arriving rays will have nearly the same LOS departing the aircraft. On this account we may write:

$$
h_{k, n} \approx e^{j 2 \pi n T_{s} V_{t} \cdot L_{t, k} / \lambda_{c}} \sum_{m \in \dot{M}(k, n)} \alpha_{m}^{\prime} e^{j 2 \pi n T_{s} V_{r} \cdot L_{r, m} / \lambda_{c}}
$$

since

$$
L_{t, k} \approx L_{t, m} \quad \forall m \in M^{\prime}(k, n)
$$

At this time some observations are in order. Since the channel response described in (8) is generally applicable, if we apply it in the case of the AtG channel we will observe that the doppler bandwidth of this channel is quite 
extreme in comparison to typical GtG scenarios. Indeed, for realistic vehicle mobility assumptions, the fading rate is dominated by the doppler induced by the high aircraft velocity. Consequently, an adaptation to such channel behavior would be quite demanding.

However, in (9) we note that the fading rate of the summation is comparable with typical GtG scenarios since it is based purely on ground vehicle mobility. This is modified by a delay-dependent phase rotation entirely due to aircraft mobility. This observation leads to the following time-variant channel model which we call the AtG multipath model (AtGMM):

$$
h_{k, n} \approx e^{j \omega_{k} n T_{s}} h_{k}
$$

where:

$$
h_{k} \equiv \sum_{m \in M(k, n)} \alpha_{m}^{\prime} e^{j 2 \pi n T_{s} V_{r} \cdot L_{r, m} / \lambda_{c}}
$$

and

$$
\omega_{k} \equiv 2 \pi V_{t} \cdot L_{t, k} / \lambda_{c}
$$

This channel model is depicted in Fig. 4. For our AtG model we see that the fading rate is similar to that of the GtG model when the deterministic phase rate is known.

\section{OPTIMAL EQUALIZATION UNDER HIGH MOBILITY}

Given this new model for the AtG case the next question we must consider is how to best exploit it. This model holds that doppler of all rays are not the same. If the doppler "dispersion", viz. the doppler spread, of the different multipaths is large enough then, from previous research [8, we know that significant ICI will result. In section 4 we seek to quantify this effect, but for the present we will consider an approach to exploiting the AtGMM to mitigate the effect.

\subsection{AtGMM Channel Estimation}

From (??) the channel parameters we would like to estimate are the complex coefficients $h_{n, k}$, and the associated frequencies $\omega_{k}$. Since the system is dynamical, conventional adaptive filters are generally not appropriate and we will be forced to consider a Kalman filter. Further, since this system is non-linear, we should consider the "extended" variant of the Kalman filter (EKF). The system we wish to model has the following state propagation and observation equations, respectively:

$$
\begin{aligned}
x_{n+1} & =f_{n}\left(x_{n}\right)+g_{n}\left(x_{n}\right) u_{n} \\
y_{n} & =h_{n}\left(x_{n}\right)+\nu_{n}
\end{aligned}
$$

In the following, we take the the states to be estimated as random constants and hence we assume $u_{n}=0$.

The EKF for estimating $x_{n}$ is described by the familiar expressions below 9 :

$$
\begin{aligned}
\hat{x}_{n+1 \mid n} & =f_{n}\left(\hat{x}_{n \mid n}\right) \\
\hat{x}_{n \mid n} & =\hat{x}_{n \mid n-1}+K_{f, n}\left[y_{n}-h_{n}\left(\hat{x}_{n \mid n-1}\right)\right] \\
K_{f, n} & =P_{n \mid n-1} H_{n}^{*}\left(H_{n} P_{n \mid n-1} H_{n}^{*}+R_{n}\right)^{-1} \\
P_{n \mid n} & =\left(I-K_{f, n} H_{n}\right) P_{n \mid n-1} \\
P_{n+1 \mid n} & =F_{n} P_{n \mid n} F_{n}^{*}+G_{n} Q G_{n}^{*}
\end{aligned}
$$

where:

$$
\begin{aligned}
F_{n} & =\left.\frac{\partial f_{n}(x)}{\partial x}\right|_{x=\hat{x}_{n \mid n}} \\
H_{n} & =\left.\frac{\partial h_{n}(x)}{\partial x}\right|_{x=\hat{x}_{n \mid n}}
\end{aligned}
$$


and $Q=0$ on account of the previous assumption. For the AtGMM we define the following state vector:

$$
x_{n} \equiv\left[\begin{array}{lllllll}
h_{0, n} & e^{j \omega_{0} T_{s}} & h_{1, n} & e^{j \omega_{1} T_{s}} & \cdots & h_{L-1, n} & e^{j \omega_{L-1} T_{s}}
\end{array}\right]^{T}
$$

where $h_{k, n}$ and $e^{j \omega_{k} T_{s}}$ are those in (10). Our state propagation function $f_{k, n}\left(x_{n}\right)$ is time-invariant and is uncoupled between different tap delays as follows:

$$
x_{n+1, k} \equiv\left[\begin{array}{ll}
h_{k, n+1} & e^{j \omega_{k} T_{s}}
\end{array}\right]^{T}=\left[\begin{array}{ll}
h_{k, n} e^{j \omega_{k} T_{s}} & e^{j \omega_{k} T_{s}}
\end{array}\right]^{T}
$$

where:

$$
x_{n} \equiv\left[\begin{array}{llll}
x_{n, 0}^{T} & x_{n, 1}^{T} & \cdots & x_{n, L-1}^{T}
\end{array}\right]^{T}
$$

The observation equation above is the cyclic convolution of the input vectors $s_{i}$ and the time-variant channel $h_{n}$ and is obtained as the individual rows taken from (2) (with $C_{i}$ in place of $C$ ) so that:

$$
y_{n}=Y_{i, m}=\sum_{k=0}^{L-1} h_{k, n} s_{i, j}+\nu_{n}
$$

where $s_{i, k}$ is the $k^{t h}$ element of $s_{i}, m=[n \bmod (N+L)]-L$, and $j=(N-k+m) \bmod N$. The expression for $m$ accounts for the fact that only $N$ of every $N+L$ observations are processed in channel estimation.

Again, because the states corresponding to different tap delays are uncoupled, we can express $F_{n}$ and $H_{n}$ in terms of a specific delay $k$ :

$$
\begin{aligned}
F_{n, k} & =\left[\begin{array}{cc}
e^{j \omega_{k} T_{s}} & h_{k, n} \\
0 & 1
\end{array}\right] \\
H_{n, k} & =\left[\begin{array}{ll}
s_{i, j} & 0
\end{array}\right]
\end{aligned}
$$

with the complete matrices expressed as:

$$
\begin{aligned}
F_{n} & =\operatorname{diag}\left(\left[\begin{array}{lllll}
F_{n, 0} & F_{n, 1} & \cdots & F_{n, L-1}
\end{array}\right]\right) \\
H_{n} & =\left[\begin{array}{llll}
H_{n, 0} & H_{n, 1} & \cdots & H_{n, L-1}
\end{array}\right]
\end{aligned}
$$

In section 4.3 we present a comparison of the signal-to-interference-ratio performance using this channel estimationbased method with that of conventional OFDM equalization.

\section{PERFORMANCE OF CONVENTIONAL-OFDM IN THE HIGH-MOBILITY ENVIRONMENT}

Previously, we have developed a model for the AtG channel environment as well as a means of channel estimation. Because the channel estimation-based optimal equalization method uses the matrix inverse of $C_{i}$ it is straightforward to see that this method eliminates ICI (at substantial complexity cost). However, when conventional equalization is employed we know that ICI is unavoidable. In this section we consider the performance implications of the AtGMM for conventional equalization.

\subsection{Computing the Average SINR for the AtGMM}

Since the frequency offset of each delay within the AtGMM is different, we can employ existing analyses [8] of carrier-offset performance as a starting point. In our analysis we consider the average SINR across the entire channel bandwidth since, in the AtGMM, we are dealing with a frequency-selective channel. Furthermore, since we expect to code our information across multiple subchannels, the SINR we are interested in can be expressed as the ratio of the average signal energy to the average noise+interference energy. Finally we note that perfect channel knowledge is assumed in the following analysis. Clearly, practical systems must employ channel estimation of some sort and, consequently, additional degradation must be expected. The effects of imperfect channel knowledge are covered in [10]. 
We can evaluate the average SINR across the subchannels as the ratio of the average received "useful" energy to the average noise plus self-interference energy:

$$
S I N R=\frac{\bar{S}}{N_{o}+\bar{A}}
$$

where $\bar{S} \equiv \frac{1}{N} \sum S_{n}$ and $\bar{A} \equiv \frac{1}{N} \sum A_{n}$.

Starting from [8, the interference to the $n^{t h}$ subcarrier due to the $i^{t h}$ subcarrier and the $k^{t h}$ multipath in the AtGMM environment is:

$$
\begin{aligned}
I_{n, i, k} & =\frac{1}{\sqrt{N}} \sum_{m=0}^{N-1} h_{k, m} e^{-j 2 \pi(i k+i m-n m) / N} \\
& =\frac{1}{\sqrt{N}} \sum_{m=0}^{N-1} h_{k} e^{j \omega_{k}(m+M) T_{s}} e^{-j 2 \pi(i k+i m-n m) / N}
\end{aligned}
$$

where $M$ is an integer multiple of $K$ and the "useful" signal within the $n^{\text {th }}$ subcarrier due to the $k^{\text {th }}$ multipath is $I_{n, n, k}$. The total signal energy within the $n^{t h}$ subcarrier results from the coherent sum due to all contributing multipaths:

$$
S_{n}=\left|\sum_{k=0}^{g-1} I_{n, n, k}\right|^{2}
$$

The total interference energy within the $n^{\text {th }}$ subcarrier results from the coherent sum due to all contributing multipaths from all subcarriers $i \neq n$ :

$$
A_{n}=\sum_{i \neq n}\left|\sum_{k=0}^{g-1} I_{n, i, k}\right|^{2}
$$

Also, it can be shown that the average interference power per subcarrier may be calculated as:

$$
\bar{A}=\|h\|^{2}-\bar{S}
$$

with

$$
\bar{S}=\sum_{k=0}^{g-1}\left|h_{k}\right|^{2} \frac{\sin ^{2}\left(\omega_{k} N / 2\right)}{N^{2} \sin ^{2}\left(\omega_{k} / 2\right)}
$$

The previous two expressions provide the necessary information to calculate the desired SINR.

\subsection{Minimizing ICI in the AtGMM}

OFDM systems commonly employ carrier synchronization to mitigate the ICI that would otherwise result without it. Ideally, we would like to maximize the average SINR as described. This may be done by, equivalently, minimizing the ICI or maximizing the average signal power. The result is more immediate if we choose the latter. The average signal power with carrier synchronization is given as:

$$
\bar{S}=\sum_{k=0}^{g-1}\left|h_{k}\right|^{2} \frac{\sin ^{2}\left(\left(\omega_{k}-\omega\right) N / 2\right)}{N^{2} \sin ^{2}\left(\left(\omega_{k}-\omega\right) / 2\right)}
$$

where $\omega$ is the carrier synchronization frequency. Maximizing (15) by finding the $\omega$ that zeros its derivative yields an intractable expression that may only evaluated by numerical methods. Alternatively, we can approximate this if we recognize that the individual terms in (15) are all maximized when $\omega=\omega_{k}$ for their respective $\omega_{k}$ 's. Furthermore, we recognize that the terms are minimized at the same set of points. And, invoking a small-angle approximation, we arrive at the following expression to be minimized:

$$
\sum_{k=0}^{g-1}\left|h_{k}\right|^{2}\left(\omega_{k}-\omega\right)^{2}
$$


which is clearly minimized when:

$$
\omega=\frac{\sum_{k=0}^{g-1} \omega_{k}\left|h_{k}\right|^{2}}{\|h\|^{2}}
$$

Viz., we can minimize the ICI by driving the carrier synchronization frequency to be equal to the power-weighted average doppler; an intuitively satisfying result. Although (16) only approximately maximizes (15), for even a moderately-dispersive set of doppler components this result is sufficiently accurate to assure a small signal loss when compared to using the optimal carrier frequency.

\subsection{Simulation Results}

In Fig. 5 we present simulation results which compare the simulated and theoretical SINR for a particular selection of tap coefficients for an input SNR of 30dB. A 64-subcarrier system with 16 sample cyclic prefix is simulated. The comparisons include the theoretical SINR of Conventional OFDM (Eq. (14)), simulated SINR of Conventional OFDM (Sim COFDM), and simulated SINR of Optimal OFDM (Sim OOFDM). For these comparison cases we use the following vector of tap coefficients: $\{0.8-0.1 j, 0.1-0.3 j,-0.1+0.05 j\}$, all other taps are zero. The time/frequency-dispersive case uses the following set of phase rotations to propagate the phase of each tap: $\left\{e^{j 3 \omega}, e^{-j 2 \omega}, e^{j 0.5 \omega}\right\}$ where we vary $\omega$ to cover the range of RMS frequency offsets of interest. Because of the frequency-dispersion, we characterize the normalized frequency offset as follows:

$$
\epsilon \equiv \frac{N}{2 \pi} \omega_{R M S}
$$

where

$$
\omega_{R M S} \equiv\left(\frac{1}{|h|^{2}} \sum_{k=0}^{g-1}\left|h_{k}\right|^{2} \omega_{k}^{2}\right)^{1 / 2}
$$

This represents the weighted RMS frequency offset to subcarrier bandwidth ratio.

We note that for a normalized frequency offset exceeding 1\%, that significant loss in SINR occurs in conventional systems. However, as we noted in section 3, the optimal OFDM processing is able to eliminate ICI. Consequently, we can maintain the input SNR regardless of the various tap frequency offsets. We also note that these results assume that the frequency offsets for each tap are within $1 / 2$ the subcarrier bandwidth.

\section{THROUGHPUT OPTIMIZATION FOR CONVENTIONAL-OFDM IN THE HIGH-MOBILITY ENVIRONMENT}

In the previous section we derived an expression for the SINR within the AtGMM context when conventional OFDM is used. This allows us to assess the effect on the effective SNR, viz. the SINR, when confronted with a specific time/frequency dispersive scenario. To asses this effect, we note that it is necessary to consider both the number of subcarriers to be used as well as the specific RMS frequency offset. They must be jointly considered because, as we see in (15), $N$ may be traded against the specific set of $\omega_{k}$ 's (in addition to the proper choice for the carrier synchronization frequency $\omega$ ). This suggests that under certain severe situations we may wish to decrease $N$ to a point where the ICI is not problematic. However, since OFDM is burdened with the overhead of a cyclic prefix, this strategy will reduce the information throughput. To put this more precisely, the information throughput can be expressed:

$$
\eta=\frac{N}{N+L} b
$$

where $b$ is the number of bits per subcarrier and the throughput, $\eta$, is expressed in units of bits/sample.

In order to fairly compare various options, it is necessary to establish a specific (uncoded) BER performance criterion to be applied to all options. Consequently, this BER criterion has been established for all results in this section at $10^{-3}$. Under this requirement, we note that the number of bits per subcarrier $b$ has a nearly linear relationship to SNR per bit (expressed in $\mathrm{dB}$ ) and $b^{2}$ also has a linear relationship to SNR. Taking the logarithm of (14) we can extract $b$ and use it in (17). We will then have an expression we can use to optimize the selection of $N$ to maximize the information throughput. 
As an example, we have shown in Fig. 6] several throughput curves corresponding to different mobility scenarios. The three scenarios correspond to stationary, low-dynamic, and high-dynamic mobility and use the same time/frequncy dispersive channel tap parameters used earlier and having RMS frequency offsets of $10^{-5}$, $10^{-4}$, and $10^{-3}$ cycles per sample respectively.

From Fig. 6 we realize that at on the high end, throughput is limited by frequency offset sensitivity. On the low end, throughput is limited by the cyclic prefix overhead (which is taken as $L=16$ in this section). Consequently, an optimization problem presents itself. We can now determine the optimal number of subcarriers that will maximize throughput given a particular RMS frequency offset subject to our BER constraint. Fig. 6 presents the results of this numerical analysis.

For very small RMS frequency offsets, the optimal symbol size is quite linear with change in RMS frequency offset. Then as we approach higher offsets, resulting in significant ICI, the slope decreases. This is due to the fact that as the cyclic prefix becomes a more significant proportion of the total symbol that throughput then begins to suffer. Consequently, as the frequency offset increases further, corresponding decreases in the throughputoptimal symbol size lessen when compared to those of the small-offset regime. This figure can be used in the design of conventional OFDM systems within fading contexts for selection of the appropriate symbol size.

Finally, in Fig. 8 we present the corresponding throughput that can optimally be achieved for a given RMS frequency offset.

\section{CONCLUSION}

In this paper we have addressed the performance implications of high mobility with regard to OFDM modulation. In order to study this it was useful to derive an AtGMM channel model to replace the conventional time-invariant tapped delay line model. For this new model we obtained a means to estimate the parameters using an EKF. The result of such an estimation could be applied to an estimation-based equalization within the time domain for OFDM. Recognizing that such an equalization approach is prohibitively complex we then considered the effect of the AtG mobility within the context of conventional OFDM equalization and derived a performance measure in the form of an SINR. This expression can be used to evaluate specific time/frequency dispersive AtGMM channels. Simulations were performed that confirmed correspondence with the derived theory. The simulation also supports the claim that the optimal equalization eliminates ICI. Continuing the theme of using conventional OFDM within high mobility contexts we presented numerical analysis showing that an optimal symbol size could be selected that would maximize the information-loading within each subcarrier, given a specific received SNR and BER constraint.

\section{ACKNOWLEDGMENTS}

The authors would like to thank the Office of Naval Research for their support of this research.

\section{REFERENCES}

1. S. B. Weinstein and P. M. Ebert, "Data transmission by frequency-division multiplexing using the discrete fourier transform," IEEE Trans. Commun. Technol. , pp. 628-634, Oct. 1971.

2. R. Prasad and S. Hara, "An overview of multi-carrier cdma," IEEE Commun. Mag., pp. 126-133, Dec. 1997.

3. Y. G. Li, J. H. Winters, and N. R. Sollenberger, "Mimo-ofdm for wireless communications: Signal detection with enhanced channel estimation," IEEE Trans. Commun. , pp. 1471-1477, Sept. 2002.

4. M. Schnell and S. Kaiser, "Diversity considerations for mc-cmda systems in mobile communications," in Proceedings of ISSSTA'95 International Symposium on Spread Spectrum Techniques and Applications, pp. 131135, (Mainz, Germany), Sept. 1995.

5. M. Foschini, G.J.; Gans, "On limits of wireless communications in a fading environment when using multiple antennas," Wireless Personal Communications 6, pp. 311-335, Mar. 1998.

6. J. G. Proakis, Digital Communications, ch. 14, p. 760. McGraw Hill, Boston, MA, 3 ed., 1995.

7. M. D. Yacoub, Foundation of Mobile Radio Engineering, ch. 3, p. 92. CRC Press, Boca Raton, FL, 1993. 


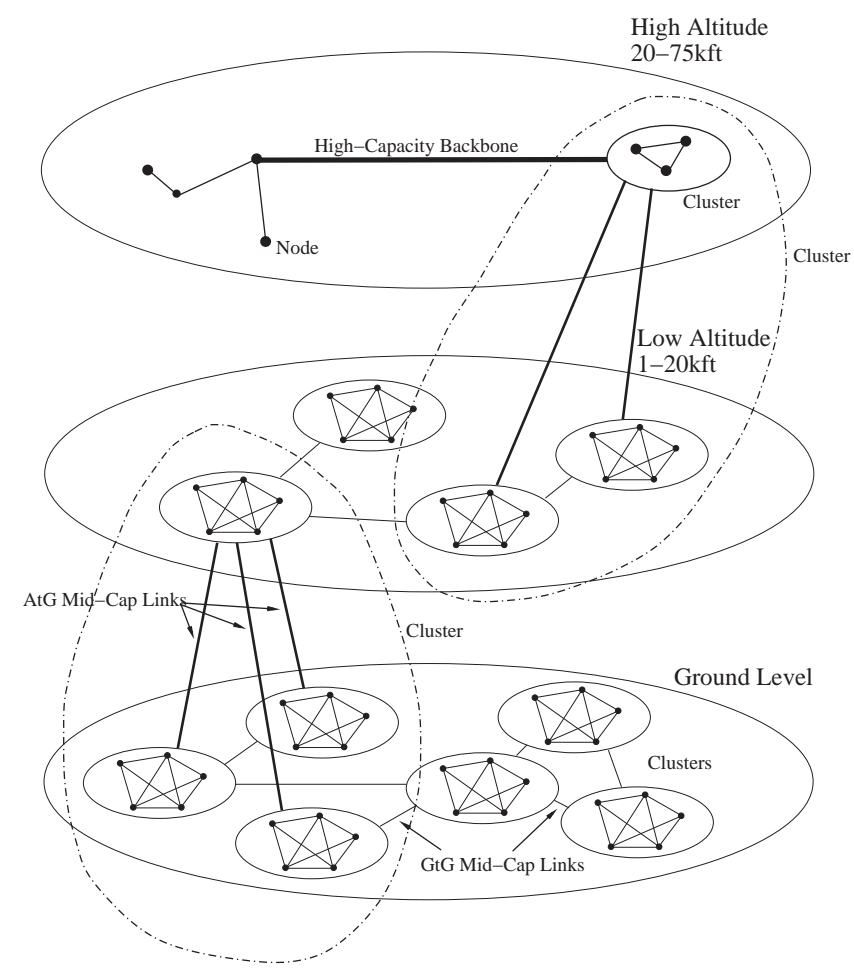

Figure 1. Hierarchical Network

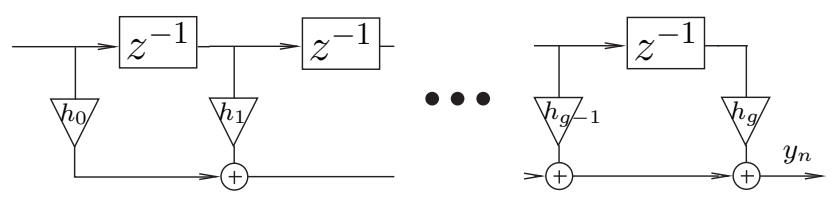

Figure 2. Conventional Time-Invariant Discrete-Time Channel Model

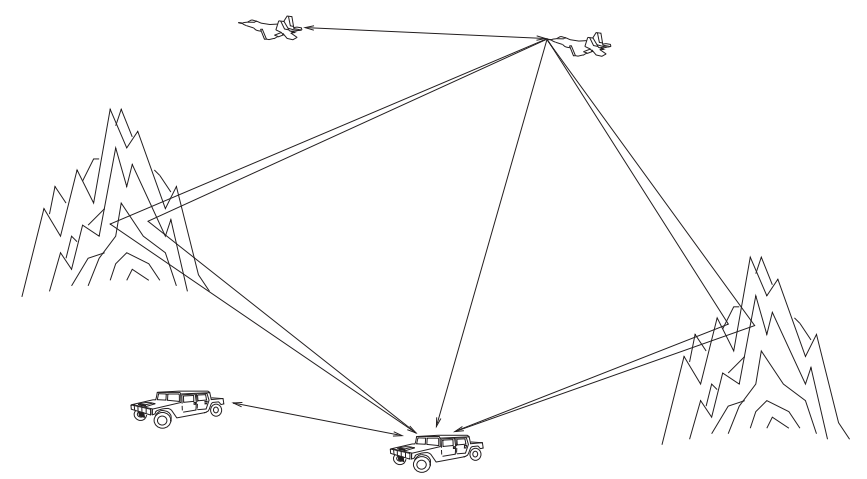

Figure 3. AtA, GtG, and AtG Multipath Environments

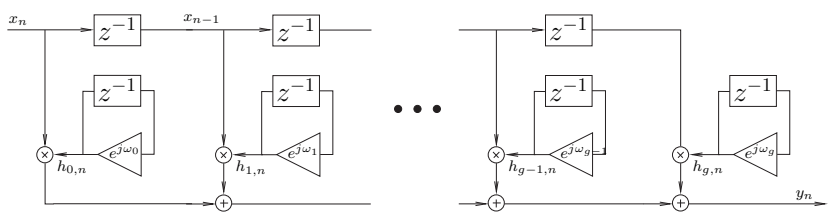

Figure 4. AtG (Time-Variant) Discrete-Time Channel Model 


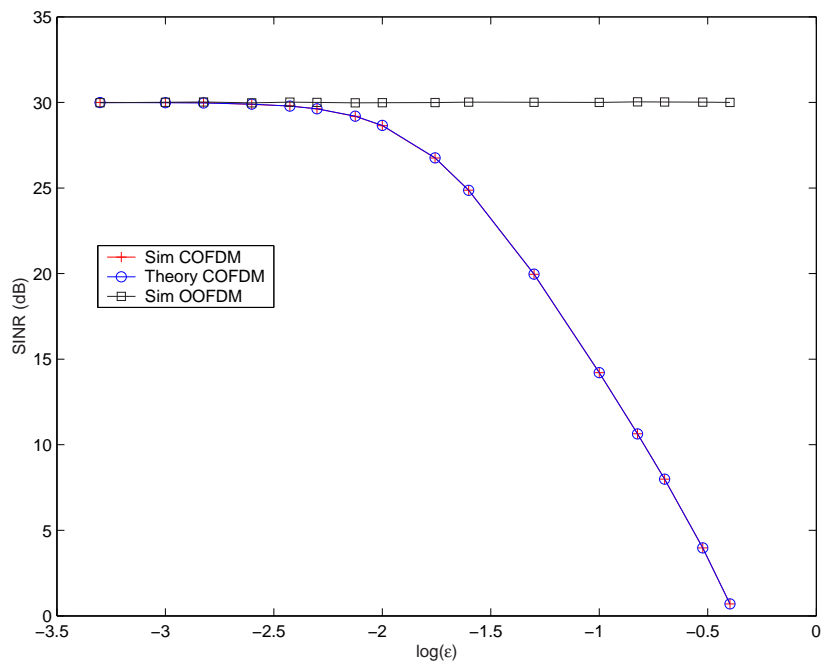

Figure 5. Comparison of Conv VS Opt OFDM Processing

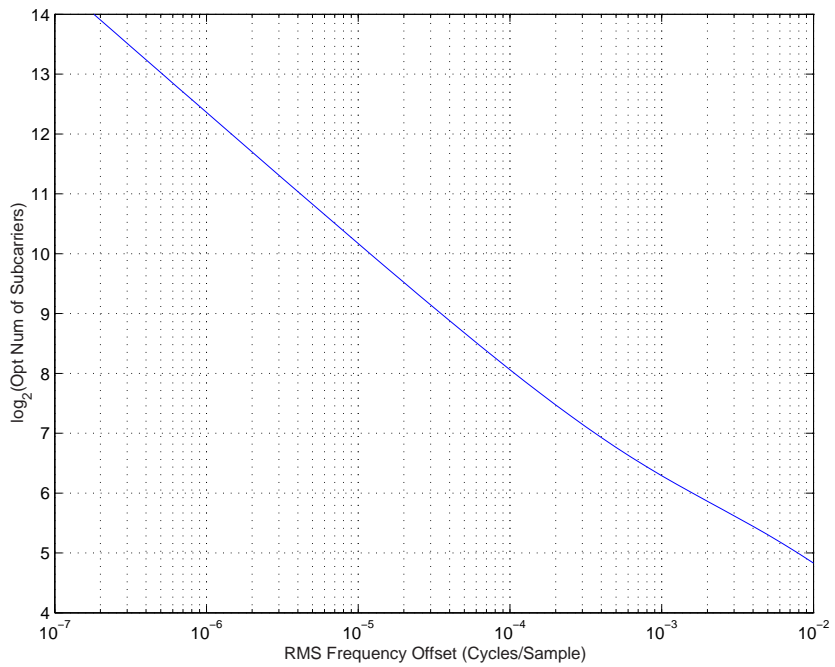

Figure 7. Optimal Symbol Size VS RMS Freq Offset

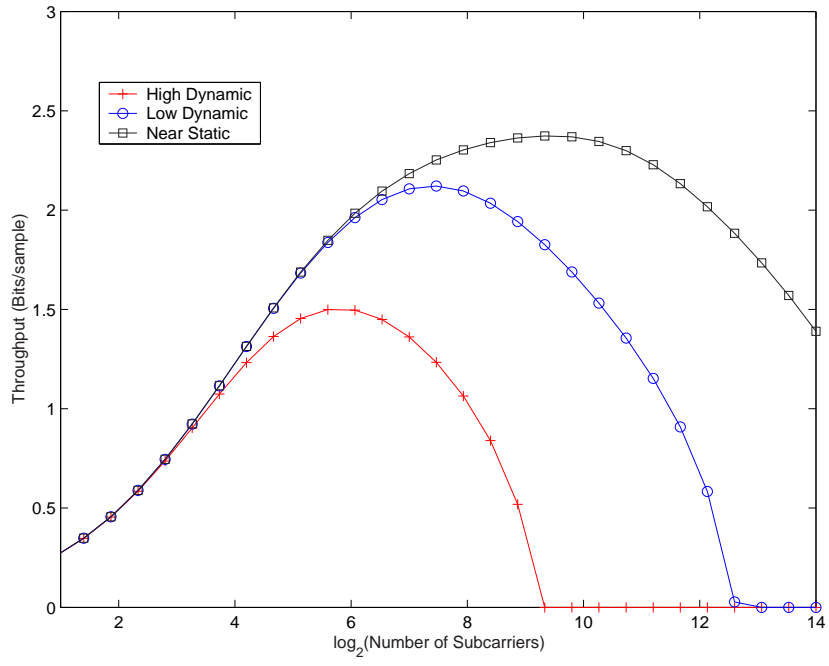

Figure 6. Example OFDM Throughput Performance

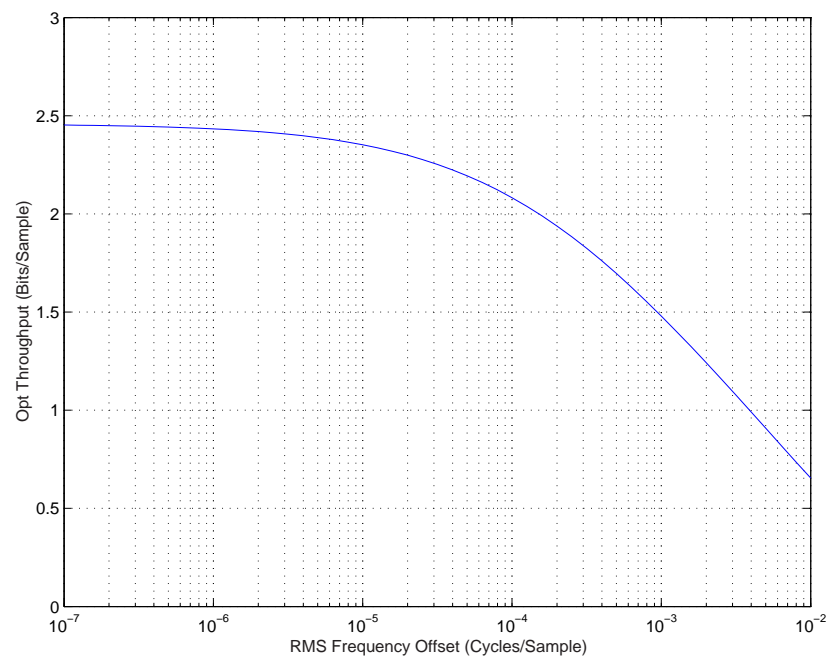

Figure 8. Optimal Throughput VS RMS Frequency Offset 
8. H. Steendam and M. Moeneclaey, "Sensitivity of orthogonal frequency-division multiplexed systems to carrier and clock synchronization errors,"

9. A. H. Sayed, Linear Estimation, ch. 9, p. 340. Prentice Hall, Upper Saddle River, NJ, 2000.

10. L. Achankeng and J. M. Cioffi, "Impact of imperfect channel knowledge on the performance of multicarrier systems," in Proc. IEEE International Conference on Communications (IEEE ICC GLOBECOM '98), 2, pp. 951-955, (Piscataway, NJ), Nov. 1998. 